\title{
Weighted endpoint estimates for multilinear commutator of singular integral operators with non-smooth kernels
}

\author{
Mingjun Zhang* and Yanfeng Guo
}

\section{"Correspondence:}

zhangmingjun2004@sina.com

College of Science, Guangxi

University of Science and

Technology, Liuzhou, 545006,

P.R. China

\begin{abstract}
In this paper, we prove the weighted endpoint estimates for multilinear commutator of singular integral operators with non-smooth kernels.
\end{abstract}

Keywords: multilinear commutator; singular integral operator; BMO

\section{Introduction}

Let $b \in B M O\left(R^{n}\right)$ and $T$ be the Calderón-Zygmund operator, the commutator $[b, T]$ generated by $b$ and $T$ is defined by $[b, T](f)(x)=b(x) T(f)(x)-T(b f)(x)$. A classical result of Coifman et al. (see [1]) proved that the commutator $[b, T]$ is bounded on $L^{p}\left(R^{n}\right)$ $(1<p<\infty)$. In [2, 3], the boundedness properties of the commutators for the extreme values of $p$ are obtained. In this paper, we will introduce the multilinear commutator of singular integral operators with non-smooth kernels and prove the weighted boundedness properties of the operator for the extreme cases.

First let us introduce some notations (see [3-12]). In this paper, $Q$ will denote a cube of $R^{n}$ with sides parallel to the axes. For a cube $Q$ and a function $b$, let $b_{Q}=|Q|^{-1} \int_{Q} b(x) d x$ and $b(Q)=\int_{Q} b(x) d x$, the sharp function of $b$ is defined by

$$
b^{\#}(x)=\sup _{Q \ni x} \frac{1}{|Q|} \int_{Q}\left|b(y)-b_{Q}\right| d y .
$$

It is well known that (see [6])

$$
b^{\#}(x)=\sup _{Q \ni x} \inf _{c \in C} \frac{1}{|Q|} \int_{Q}|b(y)-c| d y .
$$

Moreover, for a weight function $\omega$ (that is, a non-negative locally integrable function), $b$ is said to belong to $B M O(\omega)$ if $b^{\#} \in L^{\infty}(\omega)$ and define $\|b\|_{B M O(\omega)}=\left\|b^{\#}\right\|_{L^{\infty}(\omega)}$, if $\omega=1$, we denote $B M O(\omega)=B M O\left(R^{n}\right)$. It is well known that (see [11])

$$
\left\|b-b_{2^{k} Q}\right\|_{B M O} \leq C k\|b\|_{B M O}
$$

\section{Springer}

O2014 Zhang and Guo; licensee Springer. This is an Open Access article distributed under the terms of the Creative Commons Attribution License (http://creativecommons.org/licenses/by/2.0), which permits unrestricted use, distribution, and reproduction in any medium, provided the original work is properly cited. 
The $A_{p}$ weight is defined by (see [6])

$$
\begin{aligned}
A_{p} & =\left\{0<\omega \in L_{\mathrm{loc}}^{1}\left(R^{n}\right): \sup _{Q}\left(\frac{1}{|Q|} \int_{Q} \omega(x) d x\right)\left(\frac{1}{|Q|} \int_{Q} \omega(x)^{-1 /(p-1)} d x\right)^{p-1}<\infty\right\}, \\
1<p<\infty &
\end{aligned}
$$

and

$$
A_{1}=\left\{0<\omega \in L_{\mathrm{loc}}^{1}\left(R^{n}\right): \sup _{Q \ni x} \frac{1}{|Q|} \int_{Q} \omega(y) d y \leq c \omega(x) \text {, a.e. }\right\} .
$$

Definition 1 A family of operators $D_{t}, t>0$, is said to be an 'approximation to the identity' if, for every $t>0, D_{t}$ can be represented by the kernel $a_{t}(x, y)$ in the following sense:

$$
D_{t}(f)(x)=\int_{R^{n}} a_{t}(x, y) f(y) d y
$$

for every $f \in L^{p}\left(R^{n}\right)$ with $p \geq 1$, and $a_{t}(x, y)$ satisfies

$$
\left|a_{t}(x, y)\right| \leq h_{t}(x, y)=C t^{-n / 2} s\left(|x-y|^{2} / t\right),
$$

where $s$ is a positive, bounded, and decreasing function satisfying

$$
\lim _{r \rightarrow \infty} r^{n+\epsilon} s\left(r^{2}\right)=0
$$

for some $\epsilon>0$.

Definition 2 A linear operator $T$ is called the singular integral operators with nonsmooth kernels if $T$ is bounded on $L^{2}\left(R^{n}\right)$ and associated with a kernel $K(x, y)$ such that

$$
T(f)(x)=\int_{R^{n}} K(x, y) f(y) d y
$$

for every continuous function $f$ with compact support, and for almost all $x$ not in the support of $f$.

(1) There exists an 'approximation to the identity' $\left\{B_{t}, t>0\right\}$ such that $T B_{t}$ has associated kernel $k_{t}(x, y)$ and there exist $c_{1}, c_{2}>0$ so that

$$
\int_{|x-y|>c_{1} t^{1 / 2}}\left|K(x, y)-k_{t}(x, y)\right| d x \leq c_{2} \quad \text { for all } y \in R^{n} .
$$

(2) There exists an 'approximation to the identity' $\left\{A_{t}, t>0\right\}$ such that $A_{t} T$ has associated kernel $K_{t}(x, y)$ which satisfies

$$
\left|K_{t}(x, y)\right| \leq c_{4} t^{-n / 2} \quad \text { if }|x-y| \leq c_{3} t^{1 / 2}
$$

and

$$
\left|K(x, y)-K_{t}(x, y)\right| \leq c_{4} t^{\delta / 2}|x-y|^{-n-\delta} \quad \text { if }|x-y| \geq c_{3} t^{1 / 2}
$$

for some $c_{3}, c_{4}>0, \delta>0$. 
Given some locally integrable functions $b_{j}(j=1, \ldots, m)$. The multilinear operator associated to $T$ is defined by

$$
T_{b}(f)(x)=\int_{R^{n}}\left[\prod_{j=1}^{m}\left(b_{j}(x)-b_{j}(y)\right)\right] K(x, y) f(y) d y .
$$

Definition 3 Given the 'approximations to the identity' $\left\{A_{t}, t>0\right\}$ and a weight function $\omega$.

(1) The weighted $B M O$ space associated with $\left\{A_{t}, t>0\right\}$ is defined by

$$
B M O_{A}(\omega)=\left\{f \in L_{\text {loc }}^{1}\left(R^{n}\right):\|f\|_{B M O_{A}(\omega)}<\infty\right\},
$$

where

$$
\|f\|_{B M O_{A}(\omega)}=\sup _{Q} \frac{1}{\omega(Q)} \int_{Q}\left|f(x)-A_{t_{Q}}(f)(x)\right| \omega(x) d x,
$$

$t_{Q}=l(Q)^{2}$ and $l(Q)$ denotes the side length of $Q$.

(2) The weighted central $B M O$ space associated with $\left\{A_{t}, t>0\right\}$ is defined by

$$
C M O_{A}(\omega)=\left\{f \in L_{\mathrm{loc}}^{1}\left(R^{n}\right):\|f\|_{C M O_{A}(\omega)}<\infty\right\}
$$

where

$$
\|f\|_{C M O(\omega)}=\sup _{r>1} \frac{1}{\omega(Q(0, r))} \int_{Q}\left|f(x)-A_{t_{Q}} f(x)\right| \omega(x) d x,
$$

and $t_{Q}=r^{2}$.

Definition 4 Let $1<p<\infty$ and $\omega$ be a weighted function on $R^{n}$. We shall call $B_{p}(\omega)$ the space of those functions $f$ on $R^{n}$, such that

$$
\|f\|_{B_{p}(\omega)}=\sup _{r>1}[\omega(Q(0, r))]^{-1 / p}\left\|f \chi_{Q(0, r)}\right\|_{L^{p}(\omega)}<\infty
$$

For $b_{j} \in B M O\left(R^{n}\right)(j=1, \ldots, m)$, set $\|\vec{b}\|_{B M O}=\prod_{j=1}^{m}\left\|b_{j}\right\|_{B M O}$. Given a positive integer $m$ and $1 \leq j \leq m$, we denote by $C_{j}^{m}$ the family of all finite subsets $\sigma=\{\sigma(1), \ldots, \sigma(j)\}$ of $\{1, \ldots, m\}$ of $j$ different elements. For $\sigma \in C_{j}^{m}$, set $\sigma^{c}=\{1, \ldots, m\} \backslash \sigma$. For $\vec{b}=\left(b_{1}, \ldots, b_{m}\right)$ and $\sigma=\{\sigma(1), \ldots, \sigma(j)\} \in C_{j}^{m}$, set $\vec{b}_{\sigma}=\left(b_{\sigma(1)}, \ldots, b_{\sigma(j)}\right), b_{\sigma}=b_{\sigma(1)} \cdots b_{\sigma(j)}$ and $\left\|\vec{b}_{\sigma}\right\|_{B M O}=$ $\left\|b_{\sigma(1)}\right\|_{B M O} \cdots\left\|b_{\sigma(j)}\right\|_{B M O}$.

\section{Theorems and proofs}

We begin with some preliminaries lemmas.

Lemma $1([5,7])$ Let $\omega \in A_{1}, 1<p \leq \infty$, and $T$ be the singular integral operators with non-smooth kernels. Then $T$ is boundedness on $L^{p}(w)$.

Lemma 2 Let $\omega \in A_{1},\left\{A_{t}, t>0\right\}$ be an 'approximation to the identity' and $b \in B M O\left(R^{n}\right)$. 
(a) for every $f \in L^{\infty}\left(R^{n}\right), 1 \leq p<\infty$, and any cube $Q$,

$$
\left(\frac{1}{|Q|} \int_{Q}\left|A_{t_{Q}}\left(\left(b-b_{Q}\right) f\right)(y)\right|^{p} d y\right)^{1 / p} \leq C\|b\|_{B M O}\|f\|_{L^{\infty}}
$$

(b) for every $f \in B_{p}(\omega), 1 \leq r<p<\infty$, and any cube $Q$,

$$
\left(\frac{1}{\omega(Q)} \int_{Q}\left|A_{t_{Q}}\left(\left(b-b_{Q}\right) f\right)(y)\right|^{r} \omega(y) d y\right)^{1 / r} \leq C\|b\|_{B M O}\|f\|_{B_{p}(\omega)},
$$

where $t_{Q}=l(Q)^{2}$ and $l(Q)$ denotes the side length of $Q$.

Proof (a) Write

$$
\begin{aligned}
\left(\frac{1}{|Q|} \int_{Q}\left|A_{t_{Q}}\left(\left(b-b_{Q}\right) f\right)(y)\right|^{p} d y\right)^{1 / p} & \\
\leq & \left(\frac{1}{|Q|} \int_{Q} \int_{R^{n}} h_{t_{Q}}(x, y)^{p}\left|\left(b(y)-b_{Q}\right) f(y)\right|^{p} d y d x\right)^{1 / p} \\
\leq & \left(\frac{1}{|Q|} \int_{Q} \int_{2 Q} h_{t_{Q}}(x, y)^{p}\left|\left(b(y)-b_{Q}\right) f(y)\right|^{p} d y d x\right)^{1 / p} \\
& +\left(\sum_{k=1}^{\infty} \frac{1}{|Q|} \int_{Q} \int_{2^{k+1} Q \backslash 2^{k} Q} h_{t_{Q}}(x, y)^{p}\left|\left(b(y)-b_{Q}\right) f(y)\right|^{p} d y d x\right)^{1 / p} \\
= & I_{1}+I_{2} .
\end{aligned}
$$

We have, by Hölder's inequality,

$$
\begin{aligned}
I_{1} & \leq\left(\frac{C}{|Q \|| 2 Q \mid} \int_{Q} \int_{2 Q}\left|\left(b(y)-b_{Q}\right) f(y)\right|^{p} d y d x\right)^{1 / p} \\
& \leq C\|f\|_{L^{\infty}}\left(\frac{1}{|2 Q|} \int_{2 Q}\left|b(y)-b_{Q}\right|^{p} d y\right)^{1 / p} \\
& \leq C\|b\|_{B M O}\|f\|_{L^{\infty}} .
\end{aligned}
$$

For $I_{2}$, for $x \in Q$ and $y \in 2^{k+1} Q \backslash 2^{k} Q$, we have $|x-y| \geq 2^{k-1} t_{Q}$ and $h_{t_{Q}}(x, y) \leq C \frac{s\left(2^{2(k-1)}\right)}{|Q|}$. Thus

$$
\begin{aligned}
I_{2} \leq & C \sum_{k=1}^{\infty} 2^{(k-1) n} s\left(2^{2(k-1)}\right)\left(\frac{1}{\left|2^{k+1} Q\right|} \int_{2^{k+1} Q}\left|\left(b(y)-b_{Q}\right) f(y)\right|^{p} d y\right)^{1 / p} \\
\leq & C\|f\|_{L^{\infty}} \sum_{k=1}^{\infty} 2^{(k-1) n} s\left(2^{2(k-1)}\right)\left(\frac{1}{\left|2^{k+1} Q\right|} \int_{2^{k+1} Q}\left|b(y)-b_{2^{k+1} Q}\right|^{p} d y\right)^{1 / p} \\
& +C\|f\|_{L^{\infty}} \sum_{k=1}^{\infty} 2^{(k-1) n} s\left(2^{2(k-1)}\right)\left|b_{Q}-b_{2^{k+1} Q}\right| \\
\leq & C\|f\|_{L^{\infty}} \sum_{k=1}^{\infty} 2^{(k-1) n} s\left(2^{2(k-1)}\right)(k+1)\|b\|_{B M O} \\
\leq & C\|b\|_{B M O}\|f\|_{L^{\infty}},
\end{aligned}
$$


where the last inequality follows from

$$
\sum_{k=2}^{\infty} 2^{(k-1) n} s\left(2^{2(k-1)}\right)(k+1) \leq C \sum_{k=2}^{\infty} k 2^{-(k-1) \epsilon}<\infty
$$

for some $\epsilon>0$.

(b) Write

$$
\begin{aligned}
& \left(\frac{1}{\omega(Q)} \int_{Q}\left|A_{t_{Q}}\left(\left(b-b_{Q}\right) f\right)(y)\right|^{r} \omega(y) d y\right)^{1 / r} \\
& \leq\left(\frac{1}{\omega(Q)} \int_{Q} \int_{R^{n}} h_{t_{Q}}(x, y)^{r}\left|\left(b(y)-b_{Q}\right) f(y)\right|^{r} \omega(y) d y d x\right)^{1 / r} \\
& \leq\left(\frac{1}{\omega(Q)} \int_{Q} \int_{2 Q} h_{t_{Q}}(x, y)^{p}\left|\left(b(y)-b_{Q}\right) f(y)\right|^{r} \omega(y) d y d x\right)^{1 / r} \\
& \quad+\left(\sum_{k=1}^{\infty} \frac{1}{\omega(Q)} \int_{Q} \int_{2^{k+1} Q \backslash 2^{k} Q} h_{t_{Q}}(x, y)^{r}\left|\left(b(y)-b_{Q}\right) f(y)\right|^{r} \omega(y) d y d x\right)^{1 / r} \\
& =I+I I .
\end{aligned}
$$

For $I$, since $\omega \in A_{1}, \omega$ satisfies the reverse of Hölder's inequality

$$
\left(\frac{1}{|Q|} \int_{Q} \omega(x)^{q} d x\right)^{1 / q} \leq \frac{C}{|Q|} \int_{Q} \omega(x) d x
$$

for some $1<q<\infty$, and $\frac{\omega\left(Q_{2}\right)}{\left|Q_{2}\right|} \frac{\left|Q_{1}\right|}{\omega\left(Q_{1}\right)} \leq C$ for all cubes $Q_{1}, Q_{2}$ with $Q_{1} \subset Q_{2}, \omega \in A_{p / r u}$ for $1<u, v<\infty$ with $u^{\prime} v=q$ and $p>r u$ (see [6]). We have, by Hölder's inequality,

$$
\begin{aligned}
& I \leq\left(\frac{C}{\omega(Q)|Q|} \int_{Q} \int_{2 Q}\left|\left(b(y)-b_{Q}\right) f(y)\right|^{r} \omega(y) d y d x\right)^{1 / r} \\
& \leq C\left(\frac{1}{\omega(Q)} \int_{2 Q}\left|\left(b(y)-b_{Q}\right) f(y)\right|^{r} \omega(y) d y\right)^{1 / r} \\
& \leq C\left[\frac{|2 Q|}{\omega(Q)}\left(\frac{1}{|2 Q|} \int_{2 Q}\left|b(y)-b_{Q}\right|^{r u^{\prime}} \omega(y)^{u^{\prime}} d y\right)^{1 / u^{\prime}}\left(\frac{1}{|2 Q|} \int_{2 Q}|f(y)|^{r u} d y\right)^{1 / u}\right]^{1 / r} \\
& \leq C\left(\frac{|2 Q|}{\omega(Q)}\right)^{1 / r}\left(\frac{1}{|2 Q|} \int_{2 Q}\left|b(y)-b_{Q}\right|^{r u^{\prime} v^{\prime}} d y\right)^{1 / r u^{\prime} v^{\prime}}\left(\frac{1}{|2 Q|} \int_{2 Q} \omega(y)^{u^{\prime} v} d y\right)^{1 / r u^{\prime} v} \\
& \times\left(\frac{1}{|2 Q|} \int_{2 Q}|f(y)|^{r u} d y\right)^{1 / r u} \\
& \leq C\|b\|_{B M O}\left(\frac{|2 Q|}{\omega(2 Q)}\right)^{1 / r}\left(\frac{\omega(2 Q)}{|2 Q|}\right)^{1 / r}\left(\frac{1}{|2 Q|} \int_{2 Q}|f(y)|^{r u} \omega(y)^{\frac{r u}{p}} \omega(y)^{-\frac{r u}{p}} d y\right)^{1 / r u} \\
& \leq C\|b\|_{B M O}\left(\frac{1}{|2 Q|} \int_{2 Q}\left(|f(y)|^{r u} \omega(y)^{\frac{r u}{p}}\right)^{\frac{p}{r u}} d y\right)^{1 / p}\left(\frac{1}{|2 Q|} \int_{2 Q} \omega(y)^{-\frac{r u}{p} \frac{p}{p-r u}} d y\right)^{(p-r u) / p r u} \\
& \leq C\|b\|_{B M O}\left(\frac{1}{|2 Q|}\right)^{1 / p}\left\|f \chi_{2 Q}\right\|_{L^{p}(\omega)}\left(\frac{1}{|2 Q|} \int_{2 Q} \omega(y) d y\right)^{-1 / p}
\end{aligned}
$$


Chang and Gus Journal of Inequalities and Applications 2014, 2014:371

Page 6 of 17

http://www.journalofinequalitiesandapplications.com/content/2014/1/371

$$
\begin{aligned}
& \times\left[\left(\frac{1}{|2 Q|} \int_{2 Q} \omega(y) d y\right)\left(\frac{1}{|2 Q|} \int_{2 Q} \omega(y)^{-\frac{1}{r u}-1} d y\right)^{\frac{p}{r u}-1}\right]^{1 / p} \\
& \leq C\|b\|_{B M O} \omega(2 Q)^{-1 / p}\left\|f \chi_{2 Q}\right\|_{L^{p}(\omega)} \\
& \leq C\|b\|_{B M O}\|f\|_{B_{p}(\omega)} \text {; } \\
& I I \leq C\left(\frac{|Q|}{\omega(Q)}\right)^{1 / r} \sum_{k=1}^{\infty} 2^{(k-1) n} s\left(2^{2(k-1)}\right)\left(\frac{1}{\left|2^{k+1} Q\right|} \int_{2^{k+1} Q}\left|\left(b(y)-b_{Q}\right) f(y)\right|^{r} \omega(y) d y\right)^{1 / r} \\
& \leq C\left(\frac{|Q|}{\omega(Q)}\right)^{1 / r} \sum_{k=1}^{\infty} 2^{(k-1) n} s\left(2^{2(k-1)}\right)\left(\frac{1}{\left|2^{k+1} Q\right|} \int_{2^{k+1} Q}\left|b(y)-b_{2^{k+1} Q}\right|^{r u^{\prime}} \omega(y)^{u^{\prime}} d y\right)^{1 / r u^{\prime}} \\
& \times\left(\frac{1}{\left|2^{k+1} Q\right|} \int_{2^{k+1} Q}|f(y)|^{r u} d y\right)^{1 / r u} \\
& \leq C\left(\frac{|Q|}{\omega(Q)}\right)^{1 / r} \sum_{k=1}^{\infty} 2^{(k-1) n} s\left(2^{2(k-1)}\right)\left(\frac{1}{\left|2^{k+1} Q\right|} \int_{2^{k+1} Q}\left|b(y)-b_{2^{k+1} Q}\right|^{r u^{\prime} v^{\prime}} d y\right)^{1 / r u^{\prime} v^{\prime}} \\
& \times\left(\frac{1}{\left|2^{k+1} Q\right|} \int_{2^{k+1} Q} \omega(y)^{u^{\prime} v} d y\right)^{1 / r u^{\prime} v}\left(\frac{1}{\left|2^{k+1} Q\right|} \int_{2^{k+1} Q} f(y)^{r u} d y\right)^{1 / r u} \\
& \leq C\|b\|_{B M O} \sum_{k=1}^{\infty} 2^{(k-1) n} s\left(2^{2(k-1)}\right)(k+1)\left(\frac{|Q|}{\omega(Q)} \cdot \frac{\omega\left(2^{k+1} Q\right)}{\left|2^{k+1} Q\right|}\right)^{1 / r} \\
& \times\left(\frac{1}{\left|2^{k+1} Q\right|} \int_{2^{k+1} Q} f(y)^{r u} d y\right)^{1 / r u} \\
& \leq C \sum_{k=1}^{\infty} 2^{(k-1) n} s\left(2^{2(k-1)}\right)(k+1)\|b\|_{B M O} \omega\left(2^{k+1} Q\right)^{-1 / p}\left\|f \chi_{2^{k+1} Q}\right\|_{L^{p}(\omega)} \\
& \leq C\|b\|_{B M O}\|f\|_{B_{p}(w)} .
\end{aligned}
$$

This completes the proof.

Theorem 1 Let $T$ be the singular integral operators with non-smooth kernels, $\omega \in A_{1}$ and $\vec{b}=\left(b_{1}, \ldots, b_{m}\right)$ with $b_{j} \in B M O\left(R^{n}\right)$ for $1 \leq j \leq m$. Then $T_{b}$ is bounded from $L^{\infty}(\omega)$ to $B M O_{A}(\omega)$.

Proof It suffices to prove, for $f \in C_{0}^{\infty}\left(R^{n}\right)$, the following inequality holds:

$$
\frac{1}{\omega(Q)} \int_{Q}\left|T_{b}(f)(x)-A_{t_{Q}} T_{b}(f)(x)\right| \omega(x) d x \leq C\|f\|_{L^{\infty}(\omega)}
$$

We fix a cube $Q=Q\left(x_{0}, d\right)$. We decompose $f$ into $f=f_{1}+f_{2}$ with $f_{1}=f \chi_{Q}, f_{2}=f \chi_{\left(R^{n} \backslash Q\right)}$.

When $m=1$, set $\left(b_{1}\right)_{Q}=|Q|^{-1} \int_{Q} b_{1}(y) d y$, we have

$$
\begin{aligned}
T_{b_{1}}(f)(x) & =\int_{R^{n}}\left[\left(b_{1}(x)-\left(b_{1}\right)_{Q}\right)-\left(b_{1}(y)-\left(b_{1}\right)_{Q}\right)\right] K(x, y) f(y) d y \\
& =\left(b_{1}(x)-\left(b_{1}\right)_{Q}\right) \int_{R^{n}} K(x, y) f(y) d y-\int_{R^{n}}\left(b_{1}(y)-\left(b_{1}\right)_{Q}\right) K(x, y) f(y) d y
\end{aligned}
$$

and

$$
A_{t_{Q}} T_{b_{1}}(f)(x)=\left(b_{1}(x)-\left(b_{1}\right)_{Q}\right) \int_{R^{n}} K_{t}(x, y) f(y) d y-\int_{R^{n}}\left(b_{1}(y)-\left(b_{1}\right)_{Q}\right) K_{t}(x, y) f(y) d y .
$$


Chang and Gro Journal of Inequalities and Applications 2014, 2014:371

Page 7 of 17

Then

$$
\begin{aligned}
& \left|T_{b_{1}}(f)(x)-A_{t_{Q}} T_{b_{1}}(f)(x)\right| \\
& \leq\left|\left(b_{1}(x)-\left(b_{1}\right)_{Q}\right) \int_{R^{n}} K(x, y) f(y) d y\right| \\
& +\left|\int_{R^{n}}\left(b_{1}(y)-\left(b_{1}\right)_{Q}\right) K(x, y) f_{1}(y) d y\right| \\
& +\left|\left(b_{1}(x)-\left(b_{1}\right)_{Q}\right) \int_{R^{n}} K_{t}(x, y) f(y) d y\right| \\
& +\left|\int_{R^{n}}\left(b_{1}(y)-\left(b_{1}\right)_{Q}\right) K_{t}(x, y) f_{1}(y) d y\right| \\
& +\left|\int_{R^{n}}\left(b_{1}(y)-\left(b_{1}\right)_{Q}\right)\left(K(x, y)-K_{t}(x, y)\right) f_{2}(y) d y\right| \\
& =I_{1}(x)+I_{2}(x)+I_{3}(x)+I_{4}(x)+I_{5}(x) .
\end{aligned}
$$

For $I_{1}(x)$, let $1 / p+1 / p^{\prime}=1,1 / q+1 / q^{\prime}=1$, by the reverse of Hölder's inequality with $1<q<\infty$, Lemma 1, and Hölder's inequality, we have

$$
\begin{aligned}
& \frac{1}{\omega(Q)} \int_{Q}\left|I_{1}(x)\right| \omega(x) d x \\
& \leq \frac{C}{\omega(Q)}\left(\int_{Q}\left|b_{1}(x)-\left(b_{1}\right)_{Q}\right|^{p^{\prime}} \omega(x) d x\right)^{1 / p^{\prime}}\left(\int_{R^{n}}|T(f)(x)|^{p} \omega(x) \chi_{Q}(x) d x\right)^{1 / p} \\
& \leq \frac{C}{\omega(Q)}\left(\int_{Q}\left|b_{1}(x)-\left(b_{1}\right)_{Q}\right|^{p^{\prime}} \omega(x) d x\right)^{1 / p^{\prime}}\left(\int_{R^{n}}|f(x)|^{p} \omega(x) \chi_{Q}(x) d x\right)^{1 / p} \\
& \leq \frac{C}{\omega(Q)}\left(\int_{Q}\left|b_{1}(x)-\left(b_{1}\right)_{Q}\right|^{p^{\prime}} \omega(x) d x\right)^{1 / p^{\prime}}\|f\|_{L^{\infty}(\omega)}\left(\int_{Q} \omega(x) d x\right)^{1 / p} \\
& \leq \frac{C}{\omega(Q)}\left[\left(\int_{Q}\left|b_{1}(x)-\left(b_{1}\right)_{Q}\right|^{p^{\prime} q^{\prime}} d x\right)^{1 / q^{\prime}}\left(\int_{Q} \omega(x)^{q} d x\right)^{1 / q}\right]^{1 / p^{\prime}}\|f\|_{L^{\infty}(\omega)} \omega(Q)^{1 / p} \\
& \leq C \omega(Q)^{1 / p-1}|Q|^{1 / p^{\prime}}\left\|b_{1}\right\|_{B M O}\left(\frac{1}{|Q|} \int_{Q} \omega(x)^{q} d x\right)^{1 / p^{\prime} q}\|f\|_{L^{\infty}(\omega)} \\
& \leq C\left\|b_{1}\right\|_{B M O}\|f\|_{L^{\infty}(\omega) .}
\end{aligned}
$$

For $I_{2}(x)$, taking $p>1$, by Hölder's inequality, we have

$$
\begin{aligned}
& \frac{1}{\omega(Q)} \int_{Q}\left|I_{2}(x)\right| \omega(x) d x \\
& \leq\left(\frac{1}{\omega(Q)} \int_{R^{n}}\left|T\left(\left(b_{1}-\left(b_{1}\right)_{Q}\right) f_{1}\right)(x)\right|^{p} \omega(x) \chi_{Q}(x) d x\right)^{1 / p} \\
& \quad \leq C \omega(Q)^{-1 / p}\left(\int_{R^{n}}\left|\left(b_{1}(x)-\left(b_{1}\right)_{Q}\right) f_{1}(x)\right|^{p} \omega(x) \chi_{Q}(x) d x\right)^{1 / p} \\
& \quad \leq C \omega(Q)^{-1 / p}\left[\left(\int_{Q}\left|b_{1}(x)-\left(b_{1}\right)_{Q}\right|^{p q^{\prime}} d x\right)^{1 / q^{\prime}}\left(\int_{Q}|f(x)|^{p q} \omega(x)^{q} d x\right)^{1 / q}\right]^{1 / p}
\end{aligned}
$$


Chang and Gus Journal of Inequalities and Applications 2014, 2014:371

Page 8 of 17

http://www.journalofinequalitiesandapplications.com/content/2014/1/371

$$
\begin{aligned}
& \leq C \omega(Q)^{-1 / p}\left(\int_{Q}\left|b_{1}(x)-\left(b_{1}\right)_{Q}\right|^{p q^{\prime}} d x\right)^{1 / p q^{\prime}}\left(\int_{Q}|f(x)|^{p q} \omega(x)^{q} d x\right)^{1 / p q} \\
& \leq C \omega(Q)^{-1 / p}\left(\int_{Q}\left|b_{1}(x)-\left(b_{1}\right)_{Q}\right|^{p q^{\prime}} d x\right)^{1 / p q^{\prime}}\left(\int_{Q} \omega(x)^{q} d x\right)^{1 / p q}\|f\|_{L^{\infty}(\omega)} \\
& \leq C \omega(Q)^{-1 / p}|Q|^{1 / p q^{\prime}}\left\|b_{1}\right\|_{B M O}|Q|^{1 / p q}\left(\frac{1}{|Q|} \int_{Q} \omega(x)^{q} d x\right)^{1 / p q}\|f\|_{L^{\infty}(\omega)} \\
& \leq C\left\|b_{1}\right\|_{B M O}\left(\frac{|Q|}{\omega(Q)}\right)^{1 / p}\left(\frac{1}{|Q|} \int_{Q} \omega(x) d x\right)^{1 / p}\|f\|_{L^{\infty}(\omega)} \\
& \leq C\left\|b_{1}\right\|_{B M O}\|f\|_{L^{\infty}(\omega)} .
\end{aligned}
$$

For $I_{3}(x)$ and $I_{4}(x)$, we get, for $1<p_{1}, p_{2}<\infty$ with $1 / p_{1}+1 / p_{2}+1 / q=1$,

$$
\begin{aligned}
& \frac{1}{\omega(Q)} \int_{Q}\left|I_{3}(x)\right| \omega(x) d x \\
& \leq \frac{C}{\omega(Q)} \int_{Q}\left|b_{1}(x)-\left(b_{1}\right)_{Q}\right|\left|A_{t_{Q}}(f)(x)\right| \omega(x) d x \\
& \leq C \frac{|Q|}{\omega(Q)}\left(\frac{1}{|Q|} \int_{Q}\left|b_{1}(x)-\left(b_{1}\right)_{Q}\right|^{p_{1}} d x\right)^{1 / p_{1}} \\
& \quad \times\left(\frac{1}{|Q|} \int_{Q}\left|A_{t_{Q}}(f)(x)\right|^{p_{2}} d x\right)^{1 / p_{2}}\left(\frac{1}{|Q|} \int_{Q} \omega(x)^{q} d x\right)^{1 / q} \\
& \leq C \frac{|Q|}{\omega(Q)}\left\|b_{1}\right\|_{B M O}\|f\|_{L^{\infty}(\omega)} \frac{\omega(Q)}{|Q|} \\
& \leq C\left\|b_{1}\right\|_{B M O}\|f\|_{L^{\infty}(\omega)}, \\
& \frac{1}{\omega(Q)} \int_{Q}\left|I_{4}(x)\right| \omega(x) d x \\
& \leq \frac{1}{\omega(Q)} \int_{R^{n}}\left|A_{t_{Q}}\left(\left(b_{1}-\left(b_{1}\right)_{Q}\right) f_{1}\right)(x)\right| \omega(x) d x \\
& \leq C \frac{|Q|}{\omega(Q)}\left(\left.\frac{1}{|Q|} \int_{Q}\left|A_{t_{Q}}\left(\left(b_{1}-\left(b_{1}\right)_{Q}\right) f_{1}\right)(x)\right|_{B M O}\right|^{q^{\prime}} d x\right)^{1 / q^{\prime}}\left(\frac{1}{|Q|} \int_{Q} \omega(x)_{L^{\infty}(\omega)} d x\right)^{1 / q} \\
& \quad \leq b_{1}\left\|_{B M O}\right\| f \|_{L^{\infty}(\omega)} \frac{\omega(Q)}{|Q|} \\
& \quad \leq \quad
\end{aligned}
$$

For $I_{5}(x)$, we have

$$
\begin{aligned}
I_{5}(x) & =\left|\int_{R^{n}}\left(b_{1}(y)-\left(b_{1}\right)_{Q}\right)\left(K(x, y)-K_{t}(x, y)\right) f_{2}(y) d y\right| \\
& \leq C \sum_{k=0}^{\infty} \int_{2^{k+1} Q \backslash 2^{k} Q}\left|b_{1}(y)-\left(b_{1}\right)_{Q}\right||f(y)| \frac{d^{\delta}}{\left|x_{0}-y\right|^{n+\delta}} d y \\
& \leq C \sum_{k=1}^{\infty} \frac{d^{\delta}}{\left(2^{k-1} d\right)^{n+\delta}}\left|2^{k} Q\right|\left(\frac{1}{\left|2^{k} Q\right|} \int_{2^{k} Q}|f(y)|^{p} d y\right)^{1 / p}
\end{aligned}
$$




$$
\begin{aligned}
& \times\left(\frac{1}{\left|2^{k} Q\right|} \int_{2^{k} Q}\left|b_{1}(y)-\left(b_{1}\right)_{Q}\right|^{p^{\prime}} d y\right)^{1 / p^{\prime}} \\
\leq & C \sum_{k=1}^{\infty} k^{m} 2^{-k \delta}\left\|b_{1}\right\|_{B M O}\|f\|_{L^{\infty}(\omega)} \\
\leq & C\left\|b_{1}\right\|_{B M O}\|f\|_{L^{\infty}(\omega)},
\end{aligned}
$$

so

$$
\frac{1}{\omega(Q)} \int_{Q}\left|I_{5}(x)\right| \omega(x) d x \leq C\left\|b_{1}\right\|_{B M O}\|f\|_{L^{\infty}(\omega)} .
$$

When $m>1$, set $\vec{b}_{Q}=\left(\left(b_{1}\right)_{Q}, \ldots,\left(b_{m}\right)_{Q}\right) \in R^{n}$, where $\left(b_{j}\right)_{Q}=|Q|^{-1} \int_{Q} b_{j}(y) d y, 1 \leq j \leq m$, we have

$$
\begin{aligned}
T_{b}(f)(x)= & \prod_{j=1}^{m}\left(b_{j}(x)-\left(b_{j}\right)_{Q}\right) \int_{R^{n}} K(x, y) f(y) d y \\
& +\sum_{j=1}^{m-1} \sum_{\sigma \in C_{j}^{m}}(-1)^{m-j}\left(b(x)-(b)_{Q}\right)_{\sigma} \int_{R^{n}}\left(b(y)-(b)_{Q}\right)_{\sigma^{c}} K(x, y) f(y) d y \\
& +(-1)^{m} \int_{R^{n}} \prod_{j=1}^{m}\left(b_{j}(y)-\left(b_{j}\right)_{Q}\right) K(x, y) f(y) d y
\end{aligned}
$$

and

$$
\begin{aligned}
A_{t_{Q}} T_{b}(f)(x)= & \prod_{j=1}^{m}\left(b_{j}(x)-\left(b_{j}\right)_{Q}\right) \int_{R^{n}} K_{t}(x, y) f(y) d y \\
& +\sum_{j=1}^{m-1} \sum_{\sigma \in C_{j}^{m}}(-1)^{m-j}\left(b(x)-(b)_{Q}\right)_{\sigma} \int_{R^{n}}\left(b(y)-(b)_{Q}\right)_{\sigma^{c}} K_{t}(x, y) f(y) d y \\
& +(-1)^{m} \int_{R^{n}} \prod_{j=1}^{m}\left(b_{j}(y)-\left(b_{j}\right)_{Q}\right) K_{t}(x, y) f(y) d y,
\end{aligned}
$$

then

$$
\begin{aligned}
& \left|T_{b}(f)(x)-A_{t_{Q}} T_{b}(f)(x)\right| \\
& \leq\left|\prod_{j=1}^{m}\left(b_{j}(x)-\left(b_{j}\right)_{Q}\right) \int_{R^{n}} K(x, y) f(y) d y\right| \\
& \quad+\left|\sum_{j=1}^{m-1} \sum_{\sigma \in C_{j}^{m}}\left(b(x)-(b)_{Q}\right)_{\sigma} \int_{R^{n}}\left(b(y)-(b)_{Q}\right)_{\sigma^{c}} K(x, y) f(y) d y\right| \\
& \quad+\left|\int_{R^{n}} \prod_{j=1}^{m}\left(b_{j}(y)-\left(b_{j}\right)_{Q}\right) K(x, y) f_{1}(y) d y\right| \\
& \quad+\left|\prod_{j=1}^{m}\left(b_{j}(x)-\left(b_{j}\right)_{Q}\right) \int_{R^{n}} K_{t}(x, y) f(y) d y\right|
\end{aligned}
$$




$$
\begin{aligned}
& +\left|\sum_{j=1}^{m-1} \sum_{\sigma \in C_{j}^{m}}\left(b(x)-(b)_{Q}\right)_{\sigma} \int_{R^{n}}\left(b(y)-(b)_{Q}\right)_{\sigma^{c}} K_{t}(x, y) f(y) d y\right| \\
& +\left|\int_{R^{n}} \prod_{j=1}^{m}\left(b_{j}(y)-\left(b_{j}\right)_{Q}\right) K_{t}(x, y) f_{1}(y) d y\right| \\
& +\left|\int_{R^{n}} \prod_{j=1}^{m}\left(b_{j}(y)-\left(b_{j}\right)_{Q}\right)\left(K(x, y)-K_{t}(x, y)\right) f_{2}(y) d y\right| \\
& =J_{1}(x)+J_{2}(x)+J_{3}(x)+J_{4}(x)+J_{5}(x)+J_{6}(x)+J_{7}(x) .
\end{aligned}
$$

For $J_{1}(x)$, same as $m=1$, for some $1<q<\infty$, let $1 / q_{1}+1 / q_{2}+\cdots+1 / q_{m}+1 / q=1,1 / p+1 / p^{\prime}=1$, by Hölder's inequality, and the reverse of Hölder's inequality, we get

$$
\begin{aligned}
\frac{1}{\omega(Q)} \int_{Q}\left|J_{1}(x)\right| \omega(x) d x & \\
\leq & \frac{C}{\omega(Q)}\left(\int_{Q}\left|\left(b_{1}(x)-\left(b_{1}\right)_{Q}\right) \cdots\left(b_{m}(x)-\left(b_{m}\right)_{Q}\right)\right|^{p^{\prime}} \omega(x) d x\right)^{1 / p^{\prime}} \\
& \times\left(\int_{Q}|T(f)(x)|^{p} \omega(x) d x\right)^{1 / p} \\
\leq & \frac{C}{\omega(Q)}\left(\int_{Q}\left|b_{1}(x)-\left(b_{1}\right)_{Q}\right|^{p^{\prime}} \cdots\left|b_{m}(x)-\left(b_{m}\right)_{Q}\right|^{p^{\prime}} \omega(x) d x\right)^{1 / p^{\prime}} \\
& \times\|f\|_{L^{\infty}(\omega)}\left(\int_{Q} \omega(x) d x\right)^{1 / p} \\
\leq & \frac{C}{\omega(Q)}\|f\|_{L^{\infty}(\omega)} \omega(Q)^{1 / p} \prod_{j=1}^{m}\left[\left(\int_{Q}\left|b_{j}(x)-\left(b_{j}\right)_{Q}\right|^{p^{\prime} q_{j}} d x\right)^{1 / q_{j}}\left(\int_{Q} \omega(x)^{q} d x\right)^{1 / q}\right]^{1 / p^{\prime}} \\
\leq & C\|\vec{b}\|_{B M O}\|f\|_{L^{\infty}(\omega)} \omega(Q)^{1 / p^{\prime}+1 / p-1}|Q|^{1 / p^{\prime}\left(1 / q_{1}+\cdots+1 / q_{m}+1 / q-1\right)} \\
\leq & C\|\vec{b}\|_{B M O}\|f\|_{L^{\infty}(\omega) .}
\end{aligned}
$$

For $J_{2}(x)$, by Hölder's inequality and the reverse of Hölder's inequality, we have

$$
\begin{aligned}
& \frac{1}{\omega(Q)} \int_{Q}\left|J_{2}(x)\right| \omega(x) d x \\
& \leq \sum_{j=1}^{m-1} \sum_{\sigma \in C_{j}^{m}} \frac{C}{\omega(Q)}\left(\int_{Q}\left|\left(b(x)-b_{Q}\right)_{\sigma}\right|^{p^{\prime}} \omega(x) d x\right)^{1 / p^{\prime}} \\
& \quad \times\left(\int_{Q}\left|T\left(\left(b-b_{Q}\right)_{\sigma} c f\right)(x)\right|^{p} \omega(x) d x\right)^{1 / p} \\
& \leq C \sum_{j=1}^{m-1} \sum_{\sigma \in C_{j}^{m}}\left(\frac{1}{\omega(Q)} \int_{Q}\left|\left(b(x)-b_{Q}\right)_{\sigma}\right|^{p^{\prime}} \omega(x) d x\right)^{1 / p^{\prime}} \\
& \quad \times\left(\frac{1}{\omega(Q)} \int_{Q}\left|T\left(\left(b-b_{Q}\right)_{\sigma} c f\right)(x)\right|^{p} \omega(x) d x\right)^{1 / p}
\end{aligned}
$$




$$
\begin{aligned}
\leq & C \sum_{j=1}^{m-1} \sum_{\sigma \in C_{j}^{m}} \omega(Q)^{-1 / p^{\prime}}\left[\left(\int_{Q}\left|\left(b(x)-b_{Q}\right)_{\sigma}\right|^{p^{\prime} q^{\prime}} d x\right)^{1 / q^{\prime}}\left(\int_{Q} \omega(x)^{q} d x\right)^{1 / q}\right]^{1 / p^{\prime}} \\
& \times \omega(Q)^{-1 / p}\left(\int_{R^{n}}\left|\left(b(x)-b_{Q}\right)_{\sigma} f(x)\right|^{p} \omega(x) \chi_{Q}(x) d x\right)^{1 / p} \\
\leq & C \sum_{j=1}^{m-1} \sum_{\sigma \in C_{j}^{m}} \omega(Q)^{-1 / p^{\prime}}|Q|^{1 / p^{\prime} q^{\prime}+1 / p^{\prime} q-1 / p^{\prime}} \omega(Q)^{1 / p^{\prime}}\left\|\vec{b}_{\sigma}\right\|_{B M O} \\
& \times \omega(Q)^{-1 / p}\left(\int_{Q}\left|\left(b(x)-b_{Q}\right)_{\sigma^{c}}\right|^{p q^{\prime}} d x\right)^{1 / p q^{\prime}}\left(\int_{Q}|f(x)|^{p q} \omega^{q}(x) d x\right)^{1 / p q} \\
\leq & C \sum_{j=1}^{m-1} \sum_{\sigma \in C_{j}^{m}}\left\|\vec{b}_{\sigma}\right\|_{B M O}\left\|\vec{b}_{\sigma^{c}}\right\|_{B M O}\left(\frac{|Q|}{\omega(Q)}\right)^{1 / p} \\
& \times\left(\frac{1}{|Q|} \int_{Q} \omega(x) d x\right)^{1 / p}\|f\|_{L^{\infty}(\omega)} \\
\leq & C\|\vec{b}\|_{B M O}\|f\|_{L^{\infty}(\omega) .}
\end{aligned}
$$

For $J_{3}(x)$, taking $p>1$, by the $L^{p}(\omega)$-boundedness of $T$, we have

$$
\begin{aligned}
& \frac{1}{\omega(Q)} \int_{Q}\left|J_{3}(x)\right| \omega(x) d x \\
& \quad \leq\left(\frac{1}{\omega(Q)} \int_{R^{n}}\left|T\left(\left(b_{1}-\left(b_{1}\right)_{Q}\right) \cdots\left(b_{m}-\left(b_{m}\right)_{Q}\right) f_{1}\right)(x)\right|^{p} \omega(x) d x\right)^{1 / p} \\
& \quad \leq C \omega(Q)^{-1 / p}\left(\int_{R^{n}}\left|\left(b_{1}(x)-\left(b_{1}\right)_{Q}\right) \cdots\left(b_{m}(x)-\left(b_{m}\right)_{Q}\right) f_{1}(x)\right|^{p} \omega(x) d x\right)^{1 / p} \\
& \quad \leq C \omega(Q)^{-1 / p}|Q|^{1 / p q^{\prime}}\|\vec{b}\|_{B M O}|Q|^{1 / p q}\left(\frac{1}{|Q|} \int_{Q} \omega^{q} d x\right)^{1 / p q}\|f\|_{L^{\infty}(\omega)} \\
& \quad \leq C\|\vec{b}\|_{B M O}\left(\frac{|Q|}{\omega(Q)}\right)^{1 / p}\left(\frac{\omega(Q)}{|Q|}\right)^{1 / p}\|f\|_{L^{\infty}(\omega)} \\
& \leq C\|\vec{b}\|_{B M O}\|f\|_{L^{\infty}(\omega)} .
\end{aligned}
$$

For $J_{4}(x), J_{5}(x)$, and $J_{6}(x)$, choose $1<p, q_{j}<\infty, j=1, \ldots, m$, such that $1 / p+1 / q_{1}+\cdots+1 / q_{m}+$ $1 / q$, by Lemma 2 and similar to the proofs of $J_{1}(x), J_{2}(x)$, and $J_{3}(x)$, we get

$$
\begin{aligned}
& \frac{1}{\omega(Q)} \int_{Q}\left|J_{4}(x)\right| \omega(x) d x \\
& \leq C \frac{|Q|}{\omega(Q)} \prod_{j=1}^{m}\left(\frac{1}{|Q|} \int_{Q}\left|\left(b_{j}(x)-\left(b_{j}\right)_{Q}\right)\right|^{q_{j}} d x\right)^{1 / q_{j}} \\
& \quad \times\left(\frac{1}{|Q|} \int_{Q}\left|A_{t_{Q}}(f)(x)\right|^{p} d x\right)^{1 / p}\left(\frac{1}{|Q|} \int_{Q} \omega(x)^{q} d x\right)^{1 / q} \\
& \leq C\|\vec{b}\|_{B M O}\|f\|_{L^{\infty}(\omega)}, \\
& \frac{1}{\omega(Q)} \int_{Q}\left|J_{5}(x)\right| \omega(x) d x
\end{aligned}
$$




$$
\begin{aligned}
\leq & C \frac{|Q|}{\omega(Q)} \sum_{j=1}^{m-1} \sum_{\sigma \in C_{j}^{m}}\left(\frac{1}{|Q|} \int_{Q}\left|\left(b(x)-b_{Q}\right)_{\sigma}\right|^{q^{\prime}} d x\right)^{1 / q^{\prime}} \\
& \times\left(\frac{1}{|Q|} \int_{Q}\left|A_{t_{Q}}\left(\left(b-b_{Q}\right)_{\sigma} c f\right)(x)\right|^{p} d x\right)^{1 / p}\left(\frac{1}{|Q|} \int_{Q} \omega(x)^{q} d x\right)^{1 / q} \\
\leq & C \frac{|Q|}{\omega(Q)} \sum_{j=1}^{m-1} \sum_{\sigma \in C_{j}^{m}}\left\|\vec{b}_{\sigma}\right\|_{B M O}\left\|\vec{b}_{\sigma}\right\|_{B M O}\|f\|_{L^{\infty}(\omega)} \frac{\omega(Q)}{|Q|} \\
\leq & C\|\vec{b}\|_{B M O}\|f\|_{L^{\infty}(\omega)}, \\
\frac{1}{\omega(Q)} & \int_{Q}\left|J_{6}(x)\right| \omega(x) d x \\
\leq & C \frac{|Q|}{\omega(Q)}\left(\frac{1}{|Q|} \int_{Q}\left|A_{t_{Q}}\left(\left(b_{1}-\left(b_{1}\right)_{Q}\right) \cdots\left(b_{m}-\left(b_{m}\right)_{Q}\right) f_{1}\right)(x)\right|^{q^{\prime}} d x\right)^{1 / q^{\prime}} \\
& \times\left(\frac{1}{|Q|} \int_{Q} \omega(x)^{q} d x\right)^{1 / q} \\
\leq & C\|\vec{b}\|_{B M O}\|f\|_{L^{\infty}(\omega)} .
\end{aligned}
$$

For $J_{7}(x)$, note that $|x-y| \geq d=t^{1 / 2}$, taking $1<q_{j}<\infty, j=1, \ldots, m$ such that $1 / q_{1}+\cdots+$ $1 / q_{m}+1 / r=1$, then

$$
\begin{aligned}
J_{7}(x) \leq & C \sum_{k=0}^{\infty} \int_{2^{k+1} Q \backslash 2^{k} Q} \prod_{j=1}^{m}\left|\left(b_{j}(y)-\left(b_{j}\right)_{Q}\right)\right||f(y)| \frac{d^{\delta}}{\left|x_{0}-y\right|^{n+\delta}} d y \\
\leq & C \sum_{k=1}^{\infty} \frac{d^{\delta}}{\left(2^{k-1} d\right)^{n+\delta}}\left|2^{k} Q\right|\left(\frac{1}{\left|2^{k} Q\right|} \int_{2^{k} Q}|f(y)|^{r} d y\right)^{1 / r} \\
& \times \prod_{j=1}^{m}\left(\frac{1}{\left|2^{k} Q\right|} \int_{2^{k} Q}\left|b_{j}(y)-\left(b_{j}\right)_{Q}\right|^{q_{j}} d y\right)^{1 / q_{j}} \\
\leq & C \sum_{k=1}^{\infty} 2^{-k \delta}\|f\|_{L^{\infty}(\omega)} \prod_{j=1}^{m}\left(\frac{1}{\left|2^{k} Q\right|} \int_{2^{k} Q}\left|b_{j}(y)-\left(b_{j}\right)_{Q}\right|^{q_{j}} d y\right)^{1 / q_{j}} \\
\leq & C \sum_{k=1}^{\infty} k^{m} 2^{-k \delta} \prod_{j=1}^{m}\left\|b_{j}\right\|_{B M O}\|f\|_{L^{\infty}(\omega)} \\
\leq & C\|\vec{b}\|_{B M O}\|f\|_{L^{\infty}(\omega)},
\end{aligned}
$$

so

$$
\frac{1}{\omega(Q)} \int_{Q}\left|J_{7}(x)\right| \omega(x) d x \leq C\|\vec{b}\|_{B M O}\|f\|_{L^{\infty}(\omega)}
$$

This completes the proof of Theorem 1 .

Theorem 2 Let $1<p<\infty, \omega \in A_{1}$ and $\vec{b}=\left(b_{1}, \ldots, b_{m}\right)$ with $b_{j} \in B M O\left(R^{n}\right)$ for $1 \leq j \leq m$. Then $T_{b}$ is bounded from $B_{p}(\omega)$ to $C M O_{A}(\omega)$. 
Proof It suffices to prove for $f \in C_{0}^{\infty}\left(R^{n}\right)$, the following inequality holds:

$$
\frac{1}{\omega(Q)} \int_{Q}\left|T_{b}(f)(x)-A_{t_{Q}} T_{b}(f)(x)\right| \omega(x) d x \leq C\|f\|_{B_{p}(\omega)}
$$

for any cube $Q=Q(0, d)$ with $d>1$. Fix a cube $Q=Q(0, d)$ with $d>1$. Set $f_{1}=f \chi_{Q}, f_{2}=$ $f \chi_{\left(R^{n} \backslash Q\right)}$ and $\vec{b}_{Q}=\left(\left(b_{1}\right)_{Q}, \ldots,\left(b_{m}\right)_{Q}\right) \in R^{n}$, where $\left(b_{j}\right)_{Q}=|Q|^{-1} \int_{Q}\left|b_{j}(y)\right| d y, 1 \leq j \leq m$, we have

$$
\begin{aligned}
\left|T_{b}(f)(x)-A_{t_{Q}} T_{b}(f)(x)\right| & \left|\prod_{j=1}^{m}\left(b_{j}(x)-\left(b_{j}\right)_{Q}\right) \int_{R^{n}} K(x, y) f(y) d y\right| \\
& +\left|\sum_{j=1}^{m-1} \sum_{\sigma \in C_{j}^{m}}\left(b(x)-(b)_{Q}\right)_{\sigma} \int_{R^{n}}\left(b(y)-(b)_{Q}\right)_{\sigma^{c}} K(x, y) f(y) d y\right| \\
& +\left|\int_{R^{n}} \prod_{j=1}^{m}\left(b_{j}(y)-\left(b_{j}\right)_{Q}\right) K(x, y) f_{1}(y) d y\right| \\
& +\left|\prod_{j=1}^{m}\left(b_{j}(x)-\left(b_{j}\right)_{Q}\right) \int_{R^{n}} K_{t}(x, y) f(y) d y\right| \\
& +\left|\sum_{j=1}^{m-1} \sum_{\sigma \in C_{j}^{m}}\left(b(x)-(b)_{Q}\right)_{\sigma} \int_{R^{n}}\left(b(y)-(b)_{Q}\right)_{\sigma^{c}} K_{t}(x, y) f(y) d y\right| \\
& +\left|\int_{R^{n}} \prod_{j=1}^{m}\left(b_{j}(y)-\left(b_{j}\right)_{Q}\right) K_{t}(x, y) f_{1}(y) d y\right| \\
& +\left|\int_{R^{n}} \prod_{j=1}^{m}\left(b_{j}(y)-\left(b_{j}\right)_{Q}\right)\left(K(x, y)-K_{t}(x, y)\right) f_{2}(y) d y\right| \\
= & L_{1}(x)+L_{2}(x)+L_{3}(x)+L_{4}(x)+L_{5}(x)+L_{6}(x)+L_{7}(x) .
\end{aligned}
$$

For $L_{1}(x)$, we have

$$
\begin{aligned}
& \frac{1}{\omega(Q)} \int_{Q}\left|L_{1}(x)\right| \omega(x) d x \\
& \leq \frac{C}{\omega(Q)}\left(\int_{Q}\left|\left(b_{1}(x)-\left(b_{1}\right)_{Q}\right) \cdots\left(b_{m}(x)-\left(b_{m}\right)_{Q}\right)\right|^{p^{\prime}} \omega(x) d x\right)^{1 / p^{\prime}} \\
& \quad \times\left(\int_{Q}|T(f)(x)|^{p} \omega(x) d x\right)^{1 / p} \\
& \leq \frac{C}{\omega(Q)}\left[\left(\int_{Q}\left|\left(b_{1}(x)-\left(b_{1}\right)_{Q}\right) \cdots\left(b_{m}(x)-\left(b_{m}\right)_{Q}\right)\right|^{p^{\prime} q^{\prime}} d x\right)^{1 / q^{\prime}}\left(\int_{Q} \omega(x)^{q} d x\right)^{1 / q}\right]^{1 / p^{\prime}} \\
& \quad \times\left(\int_{Q}|f(x)|^{p} \omega(x) d x\right)^{1 / p} \\
& \leq \frac{C}{\omega(Q)}|Q|^{1 / p^{\prime} q^{\prime}}\|\vec{b}\|_{B M O}|Q|^{1 / p^{\prime} q}\left(\frac{\omega(Q)}{|Q|}\right)^{1 / p^{\prime}}\left\|f \chi_{Q}\right\|_{L^{p}(\omega)}
\end{aligned}
$$




$$
\begin{aligned}
& \leq C\|\vec{b}\|_{B M O} \omega(Q)^{-1 / p}\left\|f \chi_{Q}\right\|_{L^{p}(\omega)} \\
& \leq C\|\vec{b}\|_{B M O}\|f\|_{B_{p}(\omega) .}
\end{aligned}
$$

For $L_{2}(x)$, taking $1<s, s^{\prime}<\infty$, and $1 / s+1 / s^{\prime}=1$, we have

$$
\begin{aligned}
\frac{1}{\omega(Q)} \int_{Q}\left|L_{2}(x)\right| \omega(x) d x & \\
\leq & C \sum_{j=1}^{m-1} \sum_{\sigma \in C_{j}^{m}}\left(\frac{1}{\omega(Q)} \int_{Q}\left|\left(b(x)-b_{Q}\right)_{\sigma}\right|^{s^{\prime}} \omega(x) d x\right)^{1 / s^{\prime}} \\
& \times\left(\frac{1}{\omega(Q)} \int_{Q}\left|T\left(\left(b-b_{Q}\right)_{\sigma^{c}} f\right)(x)\right|^{s} \omega(x) d x\right)^{1 / s} \\
\leq & C \sum_{j=1}^{m-1} \sum_{\sigma \in C_{j}^{m}} \omega(Q)^{-1 / s^{\prime}}\left[\left(\int_{Q}\left|\left(b(x)-b_{Q}\right)_{\sigma}\right|^{s^{\prime} q^{\prime}} d x\right)^{1 / q^{\prime}}\left(\int_{Q} \omega^{q} d x\right)^{1 / q}\right]^{1 / s^{\prime}} \\
& \times \omega(Q)^{-1 / s}\left(\int_{Q}\left|\left(b(x)-b_{Q}\right)_{\sigma^{c}} f(x)\right|^{s} \omega(x) d x\right)^{1 / s} \\
\leq & C \sum_{j=1}^{m-1} \sum_{\sigma \in C_{j}^{m}} \omega(Q)^{-1 / s^{\prime}}|Q|^{1 / s^{\prime} q^{\prime}+1 / s^{\prime} q-1 / s^{\prime}} \omega(Q)^{1 / s^{\prime}}\left\|\vec{b}_{\sigma}\right\|_{B M O} \\
& \times \omega(Q)^{-1 / s}|Q|^{1 / r s}\left\|\vec{b}_{\sigma^{c}}\right\|_{B M O}\left(\int_{Q}|f(x)|^{p} \omega(x) d x\right)^{1 / p}\left(\int_{Q} \omega(x)^{q} d x\right)^{(p-s) / p q s} \\
\leq & C \sum_{j=1}^{m-1} \sum_{\sigma \in C_{j}^{m}}\left\|\vec{b}_{\sigma}\right\|_{B M O}\left\|\vec{b}_{\sigma^{c}}\right\|_{B M O} \omega(Q)^{-1 / p}\left\|f \chi_{Q}\right\|_{L^{p}(\omega)} \\
\leq & C\|\vec{b}\|_{B M O}\|f\|_{B_{p}(\omega) .}
\end{aligned}
$$

For $L_{3}(x)$, we have

$$
\begin{aligned}
& \frac{1}{\omega(Q)} \int_{Q}\left|L_{3}(x)\right| \omega(x) d x \\
& \quad \leq C\left(\frac{1}{\omega(Q)} \int_{R^{n}}\left|T\left(\left(b_{1}-\left(b_{1}\right)_{Q}\right) \cdots\left(b_{m}-\left(b_{m}\right)_{Q}\right) f_{1}\right)(x)\right|^{s} \omega(x) d x\right)^{1 / s} \\
& \quad \leq C \omega(Q)^{-1 / s}\left(\int_{Q}\left|\left(b_{1}(x)-\left(b_{1}\right)_{Q}\right) \cdots\left(b_{m}(x)-\left(b_{m}\right)_{Q}\right) f(x)\right|^{s} \omega(x) d x\right)^{1 / s} \\
& \quad \leq C \omega(Q)^{-1 / p}\|\vec{b}\|_{B M O}\left\|f \chi_{Q}\right\|_{L^{p}(\omega)} \\
& \leq C\|\vec{b}\|_{B M O}\|f\|_{B_{p}(\omega) .}
\end{aligned}
$$

For $L_{4}(x), L_{5}(x)$, and $L_{6}(x)$, by Lemma 2 , we have

$$
\begin{aligned}
& \frac{1}{\omega(Q)} \int_{Q}\left|L_{4}(x)\right| \omega(x) d x \\
& \quad \leq C\left(\frac{1}{\omega(Q)} \int_{Q}\left|\left(b_{1}(x)-\left(b_{1}\right)_{Q}\right) \cdots\left(b_{m}(x)-\left(b_{m}\right)_{Q}\right)\right|^{s^{\prime}} \omega(x) d x\right)^{1 / s^{\prime}}
\end{aligned}
$$


Chang and Gus Journal of Inequalities and Applications 2014, 2014:371

Page 15 of 17

http://www.journalofinequalitiesandapplications.com/content/2014/1/371

$$
\begin{aligned}
& \times\left(\frac{1}{\omega(Q)} \int_{Q}\left|A_{t_{Q}}(f)(x)\right|^{s} \omega(x) d x\right)^{1 / s} \\
& \leq C\left(\frac{1}{\omega(Q)}\right)^{1 / s^{\prime}}\left[\left(\int_{Q}\left|\left(b_{1}(x)-\left(b_{1}\right)_{Q}\right) \cdots\left(b_{m}(x)-\left(b_{m}\right)_{Q}\right)\right|^{s^{\prime} q^{\prime}} d x\right)^{1 / q^{\prime}}\right. \\
& \left.\times\left(\int_{Q} \omega(x)^{q} d x\right)^{1 / q}\right]^{1 / s^{\prime}}\|f\|_{B_{p}(w)} \\
& \leq C\left(\frac{1}{\omega(Q)}\right)^{1 / s^{\prime}}|Q|^{1 / s^{\prime} q^{\prime}}\|\vec{b}\|_{B M O}|Q|^{1 / s^{\prime} q}\left(\frac{\omega(Q)}{|Q|}\right)^{1 / s^{\prime}}\|f\|_{B_{p}(w)} \\
& \leq C\|\vec{b}\|_{B M O}\|f\|_{B_{p}(\omega)} ; \\
& \frac{1}{\omega(Q)} \int_{Q}\left|L_{5}(x)\right| \omega(x) d x \\
& \leq C \sum_{j=1}^{m-1} \sum_{\sigma \in C_{j}^{m}}\left(\frac{1}{\omega(Q)} \int_{Q}\left|\left(b(x)-b_{Q}\right)_{\sigma}\right|^{s^{\prime}} \omega(x) d x\right)^{1 / s^{\prime}} \\
& \times\left(\frac{1}{\omega(Q)} \int_{Q}\left|A_{t_{Q}}\left(\left(b-b_{Q}\right)_{\sigma} f\right)(x)\right|^{s} \omega(x) d x\right)^{1 / s} \\
& \leq C \sum_{j=1}^{m-1} \sum_{\sigma \in C_{j}^{m}} \omega(Q)^{-1 / s^{\prime}}\left[\left(\int_{Q}\left|\left(b(x)-b_{Q}\right)_{\sigma}\right|^{s^{\prime} q^{\prime}} d x\right)^{1 / q^{\prime}}\left(\int_{Q} \omega^{q} d x\right)^{1 / q}\right]^{1 / s^{\prime}} \\
& \times\left\|\vec{b}_{\sigma^{c}}\right\|_{B M O}\|f\|_{B_{p}(\omega)} \\
& \leq C \sum_{j=1}^{m-1} \sum_{\sigma \in C_{j}^{m}} \omega(Q)^{-1 / s^{\prime}}|Q|^{1 / s^{\prime} q^{\prime}+1 / s^{\prime} q-1 / s^{\prime}} \omega(Q)^{1 / s^{\prime}}\left\|\vec{b}_{\sigma}\right\|_{B M O}\left\|\vec{b}_{\sigma^{c}}\right\|_{B M O}\|f\|_{B_{p}(\omega)} \\
& \leq C\left\|\vec{b}_{\sigma}\right\|_{B M O}\|f\|_{B_{p}(\omega)} ; \\
& \frac{1}{\omega(Q)} \int_{Q}\left|L_{6}(x)\right| \omega(x) d x \\
& \leq\left(\frac{1}{\omega(Q)} \int_{R^{n}}\left|A_{t_{Q}}\left(\left(b_{1}-\left(b_{1}\right)_{Q}\right) \cdots\left(b_{m}-\left(b_{m}\right)_{Q}\right) f_{1}\right)(x)\right|^{s} \omega(x) d x\right)^{1 / s} \\
& \leq C\|\vec{b}\|_{B M O}\|f\|_{B_{p}(\omega)} .
\end{aligned}
$$

For $L_{7}(x)$, note that $|x-y| \geq d=t^{1 / 2}$, taking $1<u<p$, then

$$
\begin{aligned}
L_{7}(x) \leq & C \int_{Q^{c}} \prod_{j=1}^{m}\left|b_{j}(y)-\left(b_{j}\right)_{Q}\right||f(y)| \frac{d^{\delta}}{\left|x_{0}-y\right|^{n+\delta}} d y \\
\leq & C \sum_{k=0}^{\infty} \int_{2^{k+1} Q \backslash 2^{k} Q} \prod_{j=1}^{m}\left|b_{j}(y)-\left(b_{j}\right)_{Q}\right||f(y)| \frac{d^{\delta}}{\left|x_{0}-y\right|^{n+\delta}} d y \\
\leq & C \sum_{k=1}^{\infty} \frac{d^{\delta}}{\left(2^{k-1} d\right)^{n+\delta}}\left|2^{k} Q\right|\left(\frac{1}{\left|2^{k} Q\right|} \int_{2^{k} Q}|f(y)|^{u} d y\right)^{1 / u} \\
& \times\left(\frac{1}{\left|2^{k} Q\right|} \int_{2^{k} Q} \prod_{j=1}^{m}\left|b_{j}(y)-\left(b_{j}\right)_{Q}\right|^{u^{\prime}} d y\right)^{1 / u^{\prime}}
\end{aligned}
$$




$$
\begin{aligned}
\leq & C\|\vec{b}\|_{B M O} \sum_{k=1}^{\infty} k^{m} 2^{-k \delta}\left(\frac{1}{\left|2^{k} Q\right|}\right)^{1 / u} \\
& \times\left[\left(\int_{2^{k} Q}|f(y)|^{p} \omega(y) d y\right)^{\frac{u}{p}}\left(\int_{2^{k} Q} \omega(y)^{-\frac{u}{p-u}} d y\right)^{\frac{p-u}{p}}\right]^{1 / u} \\
\leq & C\|\vec{b}\|_{B M O} \sum_{k=1}^{\infty} k^{m} 2^{-k \delta}\left(\frac{1}{\left|2^{k} Q\right|}\right)^{1 / u}\left\|f \chi_{2^{k} Q}\right\|_{L^{p}(\omega)}\left(\frac{\omega\left(2^{k} Q\right)}{\left|2^{k} Q\right|}\right)^{-1 / p}\left|2^{k} Q\right|^{\left(\frac{p}{u}-1\right) \frac{1}{p}} \\
& \times\left[\left(\frac{1}{\left|2^{k} Q\right|} \int_{2^{k} Q} \omega(y) d y\right)\left(\frac{1}{\left|2^{k} Q\right|} \int_{2^{k} Q} \omega(y)^{-\frac{1}{\underline{p}-1}} d y\right)^{\frac{p}{u}-1}\right]^{1 / p} \\
\leq & C\|\vec{b}\|_{B M O} \sum_{k=1}^{\infty} k^{m} 2^{-k \delta} \omega\left(2^{k} Q\right)^{-1 / p}\left\|f \chi_{2^{k} Q}\right\|_{L^{p}(\omega)} \\
\leq & C\|\vec{b}\|_{B M O}\|f\|_{B_{p}(\omega)},
\end{aligned}
$$

So

$$
\frac{1}{\omega(Q)} \int_{Q}\left|L_{7}(x)\right| \omega(x) d x \leq C\|\vec{b}\|_{B M O}\|f\|_{B_{p}(\omega)}
$$

This completes the proof of Theorem 2.

\section{Competing interests}

The authors declare that they have no competing interests.

\section{Authors' contributions}

The authors completed the paper, and read and approved the final manuscript.

\section{Acknowledgements}

Project was supported by the National Natural Science Foundation of China (No. 11061003).

Received: 9 February 2014 Accepted: 29 August 2014 Published: 25 Sep 2014

\section{References}

1. Coifman, R, Rochberg, R, Weiss, G: Factorization theorems for Hardy spaces in several variables. Ann. Math. 103 611-635 (1976)

2. Harboure, E, Segovia, C, Torrea, J: Boundedness of commutators of fractional and singular integrals for the extreme values of p. III. J. Math. 41, 676-700 (1997)

3. Pérez, C, Pradolini, G: Sharp weighted endpoint estimates for commutators of singular integral operators. Mich. Math. J. 49, 23-37 (2001)

4. Deng, DG, Yan, LX: Commutators of singular integral operators with non-smooth kernels. Acta Math. Sci. 25, 137-144 (2005)

5. Duong, XT, Mclntosh, A: Singular integral operators with non-smooth kernels on irregular domains. Rev. Mat. Iberoam. 15, 233-265 (1999)

6. Garcia-Cuerva, J, Rubio de Francia, JL: Weighted Norm Inequalities and Related Topics. North-Holland Mathematics Studies, vol. 116. North-Holland, Amsterdam (1985)

7. Liu, LZ: Sharp function boundedness for vector-valued multilinear singular integral operators with non-smooth kernels. J. Contemp. Math. Anal. 45, 185-196 (2010)

8. Liu, LZ: Multilinear singular integral operators on Triebel-Lizorkin and Lebesgue spaces. Bull. Malays. Math. Soc. 35, 1075-1086 (2012)

9. Martell, JM: Sharp maximal functions associated with approximations of the identity in spaces of homogeneous type and applications. Stud. Math. 161, 113-145 (2004)

10. Pérez, C, Trujillo-Gonzalez, R: Sharp weighted estimates for multilinear commutators. J. Lond. Math. Soc. 65, 672-692 (2002)

11. Stein, EM: Harmonic Analysis: Real Variable Methods, Orthogonality and Oscillatory Integrals. Princeton University Press, Princeton (1993)

12. Torchinsky, A: Real Variable Methods in Harmonic Analysis. Pure and Applied Mathematics, vol. 123. Academic Press, New York (1986) 
10.1186/1029-242X-2014-371

Cite this article as: Zhang and Guo: Weighted endpoint estimates for multilinear commutator of singular integral operators with non-smooth kernels. Journal of Inequalities and Applications 2014, 2014:371

Submit your manuscript to a SpringerOpen ${ }^{\circ}$ journal and benefit from:

- Convenient online submission

- Rigorous peer review

- Immediate publication on acceptance

Open access: articles freely available online

- High visibility within the field

- Retaining the copyright to your article

Submit your next manuscript at $\gg$ springeropen.com 